\title{
Ensino de fotojornalismo e atividades
}

\section{culturais}

Atílio Avancini ${ }^{1}$ 


\section{Resumo}

O ensino de fotojornalismo na Escola de Comunicações e Artes da Universidade de São Paulo (ECA-USP) é ferramenta pedagógica que contribui de forma efetiva para o fomento e a promoção do acesso à cultura como ação extensionista. A disciplina Elementos de fotojornalismo, parte do curso de bacharelado em Jornalismo, organizase a partir de um método teórico-prático, aliado a duas atividades de cultura e extensão: o Espaço D'Ávila e a Semana de fotojornalismo. Estas frentes pedagógicas interagem entre si e têm o potencial de estimular a cidadania. Tendo a cultura como referência, o ensino de fotojornalismo promove diálogo interdisciplinar pela valorização de reportagens, pautas criativas e estímulo reflexivo. A difusão da cultura amplia o conhecimento e desenvolve qualificação profissional. Além de revelar o que a universidade pública produz, ela valoriza a produção imagética do estudante e também aperfeiçoa a construção da normatização da extensão universitária.

\section{Palavras-chave}

Comunicação, arte, jornalismo, fotografia, fotojornalismo. 
Teaching photojournalism courses at the University of São Paulo's School of Communication and Arts (ECA-USP) provides a pedagogical tool that contributes effectively towards increased access to culture through the advocacy of university extension programs. The course entitled Elements of photojournalism, part of the Graduation Programme in Journalism, is structured around theoretical-practical methods, in conjunction with two cultural and extramural activities: Espaço D'Ávila and the Photojournalism week. These pedagogical fronts interact with each other and stimulate social awareness. Using culture as a reference, photojournalistic instruction promotes an interdisciplinary dialogue by teaching the value of news reports, of creative editorial guidelines and reflexive stimuli. The dissemination of culture broadens knowledge and develops professional qualifications. Furthermore, it reveals production within a public university, recognizes the value of student image-making and contributes to the standardization of university extension programs.

\section{Keywords}

Communication, art, journalism, photography, photojournalism. 
O artigo parte da metodologia teórica e prática realizada no contexto do ensino de fotojornalismo no bacharelado em Comunicação Social com habilitação em Jornalismo (cursos matutino e noturno) da Escola de Comunicações e Artes da Universidade de São Paulo (ECA-USP). O objetivo é iniciar o estudante no universo da produção de imagens, dentro dos princípios da valorização de reportagens, pautas criativas e estímulo reflexivo, tendo a cultura como referência.

Por acreditar na formação de outro parâmetro para a produção imagética, a disciplina Elementos de fotojornalismo busca criar condições para o desenvolvimento individual e coletivo de formas criativas da linguagem jornalística, unindo enriquecimento cultural e valorização da memória documental. O ensino articula a troca de experiências em atividades de perfis distintos, promovendo ações interdisciplinares. A meta se desenvolve no aprofundamento teórico (trabalho escrito) e prático (criação visual), cujo processo de desenvolvimento no âmbito da imagem é exercitado e estimulado. E em duas atividades de cultura e extensão: o Espaço D'Ávila e a Semana de fotojornalismo.

A experimentação, que sempre se recicla, é um dos pilares fundamentais no ensino de fotojornalismo, pois o conceito de disciplina laboratorial é base para a realização das atividades propostas. Busca-se não reproduzir o clichê midiático da produção fotojornalística-comercial: escândalo, catástrofe, morbidez, celebridade. Mas encorajar processos compartilhados, apoiar o risco no processo criativo, e contribuir para formar profissionais, pesquisadores e cidadãos.

\section{Composição em trabalhos escrito e imagético}

O referencial teórico da disciplina Elementos de fotojornalismo se fundamenta em dois autores clássicos da fotografia: Roland Barthes e Susan Sontag. Em A câmara clara (La chambre claire), lançado em 1980, Barthes apresenta três princípios organizadores e constituintes da fotografia: o fotógrafo (operator), o leitor (spectator), o referente (spectrum). A máxima de que a "fotografia é inclassificável" faz alusão aos parâmetros da linguagem escrita, cujo discurso é melhor aprofundado. 
Barthes afirma que comparar a fotografia à realidade não é certamente o melhor caminho para descobrir a sua natureza, pois não é a realidade que a fotografia expressa, mas imagens. Por outro lado, há a constante coexistência sem a qual não existe a imagem: o isto-foi (ça-a-été). Ou seja, a presença indissociável do algo fotografado - o referente -, que adere à imagem fotográfica.

Em Diante da dor dos outros (Regarding the pain of others), lançado em 2003, Susan Sontag defende o ponto de vista de que a memória da guerra é construída pelo fotojornalismo midiático, mas tudo depende da consciência do leitor em face do sofrimento das vítimas. A ensaísta norte-americana defende que a experiência jamais pode ser substituída pela representação. O fotojornalismo do tipo hard news se descortina como cenário teatral, em que a tragédia mostrada pelas imagens representa a nossa sociedade. Com o excesso deste tipo de fotografias, não sobra espaço para o desenvolvimento do questionamento crítico.

O ensino de fotojornalismo articula e integra um conjunto de iniciativas sempre em constante mudança e readaptação, reunido no que pode ser considerado um laboratório de conceitos criativos dispostos em duas etapas obrigatórias: visitação a uma exposição fotográfica na cidade de São Paulo (trabalho escrito) e ensaio fotográfico (trabalho imagético). Inclui também duas etapas optativas de cultura e extensão: o Espaço D'Ávila e a Semana de fotojornalismo. As quatro etapas possibilitam aos estudantes um lugar integrador em comunhão com os pilares da universidade pública: ensino, pesquisa e extensão.

Em sala de aula, há uma iniciação teórica à história da arte, história da fotografia, expressão estética, técnica fotográfica e tratamento de imagem. Mas há também uma busca pela ampliação do aprendizado a partir de visitas a exposições fotográficas em espaços culturais ou museus, que deve gerar a produção de uma resenha crítica redigida pelo estudante. A ideia é propiciar uma confluência entre as linguagens escrita e imagética. Este trabalho escrito é desenvolvido por textos autorais dos estudantes, contando com fotografias legendadas. 
O trabalho prático (criação visual) se desenvolve a partir de saída fotográfica na cidade, preferencialmente em São Paulo, cuja proposta é a construção de ensaio fotográfico com doze imagens sequenciadas, ampliadas em papel fotográfico, coladas em passe-partout (papel colorplus $180 \mathrm{~g} / \mathrm{m}^{2}$ ) e acondicionados em caixa. O ensaio fotográfico é editado individualmente com o professor, a partir de 36 fotografias previamente selecionadas pelo estudante. As fotografias autorais são produzidas com câmera amadora ou profissional e reveladas em processo químico ou digital. A meta é desenvolver competência de linguagem visual para a produção de fotografias, sendo que a cada semestre a temática proposta para o ensaio é diferente e inédita. Vale ressaltar, diante destas duas etapas obrigatórias, que no jornal Lance, por exemplo, o repórter escreve e fotografa suas próprias matérias.

A fotografia de rua é o gênero básico para a construção do ensaio fotográfico. O processo é inspirado nas ações do repórter fotográfico Henri Cartier-Bresson. O francês é referência teórica para produzir crônicas imagéticas, fazendo exaltar o mundo moderno, a velocidade e a civilização urbana. Uma proposta é discutir - e aplicar - como a "ferramenta" bressoniana do momento decisivo se integra ao fotojornalismo. Isto é, a captação de expressões em lugares públicos no instante preciso do fato no ápice do movimento.

Assim, incorporando elementos da narrativa jornalística, o fotojornalismo de Cartier-Bresson lida simultaneamente com o espontâneo, o fugidio, a organização estética, o senso de humor, o poético, a crítica, a denúncia, o lugar desprezado, o periférico do acontecimento. Esta linguagem é referência precursora da reportagem fotográfica para produzir fatos da atualidade em visualidades sequenciais de modo não homogêneo.

O ensino de fotojornalismo, portanto, é articulado como espaço pedagógico em que as duas etapas obrigatórias estão integradas, tendo em vista o compartilhamento de conteúdo. Sabe-se que o processo de conhecimento em relação às imagens é lento e gradual, exigindo persistência e experimentação contínua. E é dentro deste viés laboratorial que o ensino de fotojornalismo se desdobra. 
A partir de 2011, com a parceria em sala de aula do Prof. Dr. Wagner Sousa e Silva na disciplina Elementos de fotojornalismo, as etapas obrigatórias e optativas ganharam impulso, promovendo inclusive uma nova frente com o surgimento da disciplina Laboratório de fotojornalismo (CJE 0649), ligada ao Jornal do campus. Nessa atividade, ligada à prática jornalística de reportagens pautadas sobre a USP, o estudante tem a oportunidade de colocar em execução o acervo de conhecimento imagético - teórico e prático - disponibilizado. O ensino dessa disciplina, sob a responsabilidade deste colega, é concebido para ser um trabalho colaborativo, dedicado à informação visual do Jornal do campus.

\section{Planos de valorização da cultura e extensão}

As duas atividades optativas de serviços à comunidade oferecem ao estudante, em primeiro lugar, a possibilidade de tornar pública sua produção fotográfica em exposição individual ou coletiva no Espaço D'Ávila. E, em segundo lugar, a possibilidade de conhecer profissionais e pensadores do jornalismo visual em palestras e debates durante a Semana de fotojornalismo. Tal evento é anual e gratuito, realizado com o apoio da empresa estudantil Jornalismo Júnior, sediada no Departamento de Jornalismo e Editoração da ECA-USP. Vale destacar que ambas as extensões foram idealizadas e fundadas dentro do programa da disciplina Elementos de fotojornalismo, respectivamente, em 1999 e 2007.

O objetivo é tornar a atividade de cultura e extensão universitária mais acessível para que a sociedade possa usufruir das realizações da USP. Além disso, visa incentivar alunos, funcionários e a comunidade em geral a compartilhar eventos culturais. Além de contribuir com a ideia de que as unidades da USP possam reconhecer que a cultura e extensão é tão importante quanto o ensino e a pesquisa. Tais atividades possuem características diversas: a articulação entre técnica e estética, a formação de cidadãos, o caráter interdisciplinar. E não podem se resumir à prestação de serviços à comunidade, tampouco se submeterem à lógica de mercado ou assumirem posturas assistencialistas. 
Para viabilizar as duas atividades de cultura e extensão mencionadas, é preciso dominar estratégias de difusão dos produtos em questão, já que ambos têm como objeto central a fotografia. Difundir deve ser entendido como multiplicação e socialização das iniciativas de pessoa a pessoa ou pelos meios impressos e eletrônicos. Para isso, faz-se necessário a ideia da diversidade cultural e que a área de cultura e extensão universitária determine alcances ambiciosos, mas possíveis, pois não existe planejamento se não houver metas bem estabelecidas.

O Espaço D'Ávila propõe-se a acolher ensaios fotográficos de trabalhos experimentais ou acadêmicos, como os Trabalhos de Conclusão de Curso ou de Iniciação Científica. "A produção universitária ainda é muito fechada. Estamos tentando tornar pública a produção dos alunos, a fim de quebrar as fronteiras entre universidade e sociedade" (AVANCINI, 1998, p. 17). Vale destacar que o espaço fotográfico, inaugurado em 6 de maio de 1998, homenageia o Prof. Dr. Antonio Carlos D’Ávila (1955-1997), fotógrafo e professor da ECA.

\footnotetext{
As fotografias, em geral, pegam carona na escrita, o que não está certo. As exposições no Espaço D'Ávila, organizadas graças ao empenho do professor Avancini, mostram que é enorme o potencial jornalístico e pedagógico da fotografia. Pois ela tem existência própria e autônoma no jornalismo. Essa autonomia e esse potencial serão devidamente reconhecidos e incorporados no planejamento das reportagens do programa "Nossa América Latina", no âmbito da disciplina "Jornalismo e Política Internacional", de agora em diante (KUCINSKI, 2000).
}

Comoatividade decultura extensão, oEspaço D'Ávila dissemina conhecimentos que a USP produz, privilegiando a perspectiva da inclusão social de atores e lugares esquecidos pelo jornalismo tradicional. Ainda, gera um acervo fotográfico (reserva técnica), pois preserva documentos históricos relativos ao fotojornalismo. A curadoria une atividade didático-pedagógica e de pesquisa. E a sua matriz conceitual é a "solidariedade", como ensina o Ulpiano Bezerra de Menezes, professor da Faculdade de Filosofia, Letras e Ciências Humanas da USP (FFLCH-USP).

O caráter complexo e autoral da produção curatorial pode ser expresso por vários elementos, dentre eles: estética do espaço físico, seleção do fotógrafo, 
edição de imagens, montagem e desmontagem das fotografias, texto de apresentação, confecção de release, divulgação eletrônica, criação de cartazes e convites, vernissage, organização do acervo fotográfico, catalogação de artigos e publicações midiáticas, promoção de debate com o fotógrafo, manutenção de página virtual dentro do domínio da ECA (www.eca.usp.br/espacodavila).

Em 70 curadorias, destacam-se alguns nomes que expuseram no Espaço D'Ávila: Os fotógrafos Wladimir Fontes, Vera Simonetti, Clovis Loureiro Jr., Francisco Moreira da Silva (Bill), Léo Colosso, Raoni Madalena; os estudantes estrangeiros Magaly Quiroz Bátory (Peru), Marc Costa (Espanha); os funcionários Ulisses de Paula, Fernanda Torres Magalhães; e os docentes Renato Levi e Joel La Laina Sene.

\section{Foco na Semana de fotojornalismo}

A gestão administrativa da Semana de fotojornalismo é de responsabilidade da empresa estudantil Jornalismo Júnior, idealizadora do projeto, sob a coordenação dos professores Atílio Avancini e Wagner Sousa e Silva. Evidente que há sempre a cooperação financeira dos órgãos de fomento cultural, como a Pró-Reitoria de Cultura e Extensão Universitária da USP (PRCEU-USP).

A empresa Jornalismo Júnior, conhecida como Jota, foi fundada em maio de 2004, sendo o autor deste artigo o professor responsável por representá-la perante a Universidade. Ela tem por objetivo intermediar as necessidades do mercado de trabalho e ampliar a formação do estudante do curso de jornalismo por meio de projetos complementares ao fornecido pelo corpo docente. Para tanto, promove atividades jornalísticas e assessoria de imprensa, além de eventos de extensão universitária voltados à comunidade. Toda a equipe é renovada anualmente e a maioria dos calouros participam efetivamente do movimento, cujo princípio é a prática do jornalismo alternativo. O Presidente da Jornalismo Júnior (gestão 2014-2015), Gabriel Lellis, explica o sentido e a função da empresa estudantil. 
parte dos rumos da empresa é discutida e aprovada por todos. Participar da Jota é se envolver na busca por um fazer jornalístico diferenciado. A sala 33 do Departamento de Jornalismo e Editoração é mais do que uma simples sede, mas também um espaço de livre produção, no qual não existem barreiras para criar o novo ou experimentar o jornalismo. Os estudantes responsáveis por escrever os textos possuem ampla liberdade para sugerir pautas e produzir conteúdos. Essa possibilidade de criar livremente, sem a interferência de algum editor, traz uma satisfação pelo fazer jornalístico. E o amor pelo trabalho traz consigo uma consequente qualidade (LELLIS, 2014).

Uma das características da Semana de fotojornalismo é a busca pela atualização de reflexões teóricas, trazendo o que de melhor está sendo produzido, em texto e imagem, no Brasil e no estrangeiro. Citamos a vinda, através de parceria internacional, em 2009, de Philippe Dubois, da Université Sorbonne Nouvelle Paris 3. Cada ano é escolhida a interlocução de um tema. Durante cinco dias, palestras, workshops, saída e exposição fotográfica ajudam o público a analisar e compreender o uso da imagem no meio midiático, seu apelo e importância. Além de discutir a estreita relação entre técnica e linguagem e as interferências da tecnologia digital no universo fotográfico.

O núcleo de eventos da Jota organiza a tradicional Semana de Fotojornalismo. Produzida anualmente, desde 2007, a Semana tem um tema fixo e diversos profissionais do ramo são chamados para palestrar. O foco da Semana de Fotojornalismo não é apenas produzir algo relacionado ao mundo jornalístico, mas também promover um retorno à sociedade. Por essa razão, a entrada é totalmente gratuita, e é pedido aos participantes, se possível, a doação de um quilo de alimento não perecível, posteriormente repassado para instituições beneficentes (LELLIS, 2014).

Atividade de referência nacional, a Semana de fotojornalismo promove a realização de debates e difunde a produção de fotógrafos, tendo por objetivo reunir alunos de graduação, pesquisadores, professores e outros interessados em compartilhar estudos sobre o fotojornalismo. Fundamenta-se no diálogo entre diferentes tendências sobre a fotografia, tendo como premissa que o conhecimento e a informação é de fundamental importância ao profissional. 
A escolha dos palestrantes se pauta pelo aspecto humanístico e pelo trabalho social de seu trabalho. A abertura (segunda-feira) reúne professores e pesquisadores de notório saber. Os dias intermediários (terça-feira e quinta-feira) agregam fotógrafos de experiência comprovada. O meio da programação (quarta-feira) é dedicada à saída fotográfica. O encerramento (sexta-feira) conta com debate, exposição das fotografias produzidas na saída, premiação dos melhores trabalhos e coffee-break.

Vale lembrar alguns convidados de destaque das oito edições da Semana de fotojornalismo (SF). I SF (ECA-2007): Joel La Laina Sene, Cristiano Mascaro, Ronaldo Entler, João Bittar, Toni Pires, José Diório. II SF (ECA-2008): Hélio Campos Mello, Juca Martins, Emidio Luisi, Rosa Gauditano. III SF (FAU-2009): Philippe Dubois, Rubens Machado, Ismail Xavier, Thales Trigo, Cláudio Edinger, Flávio Florido, Marlene Bergamo. IV SF (FAU-2010): Adriano Escanhuela, João Roberto Ripper, Ed Viggiani, Ormuzd Alves, Iatã Cannabrava, Anderson Schneider, Luciano Candisani. V SF (FAU-2011): Helouise Costa, Mayra Rodrigues Gomes, Dirce Carrion, Caio Guatelli, José Cordeiro, Márcio Scavone. VI SF (FAU-2012): Apu Gomes, Chico Ferreira, Alan Marques, Paulo Whitaker, Rogério Ferrari, Victor Moriyama, Evandro Teixeira. VII SF (FAU-2013): Dulcília Buitoni, Inaê Coutinho, Cláudia Ferreira, Hugo Lenzi, Mônica Zarattini. VIII SF (Paço das Artes-2014): Luciano Maluly, Milton Leite, Daniel Augusto Jr, Jorge Araújo, Rubens Chaves, Moacyr Lopes Jr.

\section{Enquadramento na constante renovação}

Como fomentar a cultura e extensão no âmbito acadêmico? Primeiro, a atividade cultural não é assistencialista, e nenhum projeto adquire consistência sem a infraestrutura necessária. Segundo, a proposta institucional e o ordenamento dos eventos devem ser compreendidos e assimilados pelo público. A contenção de verbas no cenário acadêmico contemporâneo e seu enrijecimento funcional tendem a minimizar a oferta da área cultural. Uma prova disso é a Tenda Cultural Ortega y Gasset, concebida em 2013 como espaço de vanguarda na gestão da Profa. Dra. Maria Arminda Arruda à frente da Pró-Reitoria de Cultura e Extensão Universitária da USP, mas logo desativada em 2014. 
Vindo da vitrine que a USP representa para o país, aqui muito se produz que impacta o conhecimento. Vale lembrar que a difusão da cultura é parte integrante da sociedade e se constitui direito do cidadão. Entretanto, além do econômico e burocrático, há outros fatores que inibem as atividades de cunho cultural, como o modelo de avaliação docente baseado nos parâmetros numéricos da Capes, que pouco valoriza este tipo de atividade.

Universidades existem para produzir e difundir conhecimento. Corresponder a esta premissa permite integrar ensino, pesquisa científica e extensão, formando pessoas capazes de aplicar o saber. Constata-se que a universidade pública deve enfrentar esta crise, tendo como norte a noção da qualificação da atividade cultural para agilizar a sua institucionalização dentro de modos mais ágeis e competentes. Em disciplina laboratorial é fundamental a sua reinvenção em cada semestre, isto é, articular processos de constante transformação e aperfeiçoamento do método. Mas antes de tudo, os projetos de cultura e extensão devem contar com o envolvimento da criatividade e da boa vontade de professores e estudantes. 


\section{Referências}

AVANCINI, A. Atílio Avancini. São Paulo: Edusp, 2006.

AVANCINI, A. Entre gueixas e samurais. São Paulo: Edusp/IOESP, 2008.

AVANCINI, A. Depoimento a Raquel Landim. Jornal da USP, São Paulo, 11 a 17 mai. 1998, p. 17.

BARTHES, R. A câmara clara. Rio de Janeiro: Nova Fronteira, 1984.

CARTIER-BRESSON, H. El instante decisivo. In: FONCUBERTA, J (org.). Estética fotográfica. Barcelona: Blume, 1984. p. 188-201.

KUCINSKI, B. "As linhas do Equador". Espaço D'Ávila (texto de apresentação). São Paulo, out. 2000.

LELLIS, G. Depoimento a Atílio Avancini. Universidade de São Paulo, São Paulo, ago. 2014.

SONTAG, S. Diante da dor dos outros. São Paulo: Companhia das Letras, 2003. 